\title{
MORTALIDADE ENTRE OS PORTADORES DE TUBERCULOSE EM PORTO VELHO (RO)
}

\section{Tatiane Cabral Siqueira}

Acadêmica de Enfermagem. Fundação Universidade Federal de Rondônia (UNIR), Porto Velho (RO), Brasil.

\section{Rafaele Oliveira Bonfim}

Enfermeira. Mestranda no Programa de Pós-Graduação Enfermagem em Saúde Pública. Escola de Enfermagem de Ribeirão Preto (EERP), Universidade de São Paulo, Ribeirão Preto (SP), Brasil.

\section{Melisane Regina Lima Ferreira}

Acadêmica de Enfermagem. Fundação Universidade Federal de Rondônia (UNIR), Porto Velho (RO), Brasil.

\section{Nathalia Halax Orfão}

Enfermeira. Doutora Docente do Departamento de Enfermagem. Fundação Universidade Federal de Rondônia (UNIR), Porto Velho (RO), Brasil.
RESUMO: Descrever a mortalidade entre os pacientes portadores de tuberculose no município de Porto Velho (RO). Estudo epidemiológico descritivo, do tipo transversal e abordagem quantitativa. A população foi constituída por todos os registros dos portadores de tuberculose que foram a óbito durante o tratamento, entre 2010 e 2015, no município selecionado. As variáveis foram analisadas por meio de distribuição de frequência, após atender os preceitos éticos. Verificou-se no Sistema de Informação de Agravos de Notificação (SINAN) que, independente da causa do óbito, a maioria era do sexo masculino, cor/raça parda, residia na zona urbana, caso novo, forma clínica pulmonar, não etilista, não diabético, raio-X de tórax suspeito de tuberculose e não foram acompanhados pelo TDO. Observou-se similaridade entre a causa do óbito e o perfil dos doentes de tuberculose, além de divergências entre os sistemas de informação, interferindo na avaliação dos óbitos e da realidade local.

PALAVRAS-CHAVE: Tuberculose; Mortalidade; Resultado do tratamento; Sistemas de informação em saúde.

\section{MORTALITY AMONG PEOPLE WITH TUBERCULOSIS IN PORTO VELHO, BRAZIL}

ABSTRACT: Mortality among people with tuberculosis in Porto Velho RO Brazil, is analyzed by a descriptive, transversal and quantitative epidemiological study. Population comprised all registers of people with tuberculosis who died during treatment between 2010 and 2015 in the municipality above. Variables were analyzed by frequency distribution after ethical requirements were warranted. The Information System on the Notification of Diseases (SINAN) showed that most were males, brown, lived in the urban perimeter, a new case, pulmonary type, non-alcoholic, non-diabetic, with thorax X-Rays with suspicion of TB and not accompanied by TDO. Similarity was evident between the cause of death, profile of people with TB and divergence between the information systems, which interfered in the evaluation of deaths and local reality.

KEY WORDS: Tuberculosis; Mortality; Treatment outcome; Health information systems.

\section{INTRODUÇÃO}

A tuberculose (TB) é um problema de saúde pública mundial que está diretamente relacionado às precárias condições socioeconômicas. Estima-se que, em 2015, 10,4 milhões de pessoas adoeceram 
pelo Mycobacterium tuberculosis em todo o mundo, o que causou 1,4 milhão de óbitos por TB e mais 0,4 milhões de óbitos pela coinfecção TB/HIV. Mesmo com a redução de 22\% no coeficiente de mortalidade (2000-2015), a Organização Mundial da Saúde (OMS) reconhece a TB como a doença infecciosa que causa o maior número de óbitos, superando o HIV/aids e a malária juntos ${ }^{1}$.

Em 2014, a OMS aprovou a Estratégia pelo Fim da TB, tendo como visão "Um mundo livre da TB: zero morte, adoecimento e sofrimento devido à TB", e objetivo o "fim da epidemia global da doença". Esta estabeleceu como metas: reduzir o coeficiente de incidência para menos de dez casos a cada 100 mil habitantes, bem como o número de óbitos por TB em 95\% até o ano de 2035, comparado com o ano de $2015^{2}$.

A vigilância epidemiológica utiliza o Sistema de Informação de Agravos de Notificação (SINAN) como principal ferramenta para o monitoramento da TB no Brasil. Mesmo assim, sendo o óbito um desfecho alarmante, o Sistema de Informação sobre Mortalidade (SIM) permite traçar indicadores que contribuem com a organização dos serviços, a partir da causa do óbito 3 .

A mortalidade é o desfecho que questiona a efetividade das estratégias de prevenção e controle da doença ${ }^{4}$. Sabe-se que o óbito em portadores de TB ocorre pela doença ou por não TB (NTB), como fatores externos, por exemplo. Cada óbito representa o desfecho de um conjunto de falhas dentro do sistema de saúde, bem como da sociedade, já que esse evento deveria ser evitável, considerando que a TB é uma doença tratável e curável com medicamentos de baixo custo ${ }^{5}$.

O país ocupa a $18^{\mathrm{a}}$ posição em carga de $\mathrm{TB}$, o que representa $0,9 \%$ dos casos estimados no mundo e 33\% nas Américas. Em 2015, foram notificados 75.526 casos e 4.543 óbitos por TB no país, que ocorreram principalmente nas capitais $(38,08 \%)$. Mesmo elevados, o coeficiente de incidência da doença reduziu 20,2\% entre 2006 e 2015 , e o coeficiente de mortalidade $15,4 \%$, chegando a 30,9 e 2,2 casos em 100 mil habitantes, respectivamente. Em relação aos desfechos do tratamento, observou-se baixa taxa de cura $(71,9 \%)$ e elevada de abandono $(10,4 \%)^{6,7}$.

No Estado de Rondônia, o município de Porto Velho é considerado prioritário para o Programa Nacional de Controle da Tuberculose (PNCT), onde as ações de controle da TB requerem um processo de intensificação ${ }^{8}$. Em 2015, Porto Velho apresentou um coeficiente de incidência da TB de 51,3 casos e mortalidade de 2,0 casos, ambos para cada 100 mil habitantes. Quanto aos desfechos do tratamento, verificou-se baixa taxa de cura $(70,5 \%)$ e elevada para o abandono $(15,7 \%)^{6,7}$.

Tais encerramentos indicam possíveis reflexões sobre o sistema de saúde, o que justifica este estudo, tendo em vista que o óbito é comumente associado à quimioterapia irregular, retardo no diagnóstico, presença de comorbidades, principalmente da coinfecção TB/HIV, TB droga resistente, fatores sociodemográficos e econômicos, sendo considerado um bom indicador da qualidade do controle da doença ${ }^{5}$, diante da alta taxa de mortalidade entre estes, permitindo avaliar as deficiências das ações de vigilância em saúde considerando a realidade local.

Neste sentido, o estudo teve como objetivo descrever a mortalidade entre os pacientes portadores de tuberculose que evoluíram para o óbito no município de Porto Velho-RO.

\section{METODOLOGIA}

Trata-se de um estudo epidemiológico descritivo, do tipo transversal e abordagem quantitativa.

A atenção a TB em Porto Velho é descentralizada para as unidades da Atenção Primária à Saúde (APS), as quais são responsáveis por realizar as ações de vigilância e controle da doença. O município conta ainda com uma rede de apoio, tais como laboratório, acompanhamento de TB extrapulmonar, coinfecção TB/HIV e internação, se necessário.

A população do estudo foi constituída por todos os registros dos portadores de TB que foram a óbito durante o tratamento da doença, no período de 2010 a 2015. Como critérios de inclusão considerou-se aqueles que foram notificados e que evoluíram para o óbito em Porto Velho, ainda que o tratamento tenha sido realizado em outro município.

As variáveis estudadas foram categorizadas em causa do óbito (TB e NTB), sociodemográficas (idade, sexo, raça/cor, escolaridade, zona de residência) e clínicas (tipo de caso, forma clínica, doenças e agravos associados (aids, etilismo, tabagismo e diabetes), exames diagnósticos, TDO, exames de controle mensal e tempo de tratamento). 
Os dados foram coletados no SINAN e relacionados aos dados do SIM, disponibilizados pela Secretaria Municipal de Saúde (SEMUSA). Estes, foram analisados a partir do software Statistic 13.0, da Statsoft, por meio de distribuição de frequência.

Atendendo as recomendações da resolução 466/12 do Conselho Nacional de Saúde 9 , o projeto matriz intitulado "Avaliação da situação epidemiológica da tuberculose no município de Porto Velho - RO" foi aprovado pelo Comitê de Ética em Pesquisa da UNIR, conforme número de parecer 2.399.327 CEP/NUSAU/UNIR.

\section{RESULTADOS}

No período de 2010 a 2015, foram notificados no SINAN 2.627 casos de TB no município de Porto Ve- lho, dos quais 59 (2,2\%) tiveram como desfecho o óbito, seja por outras causas (33 casos - 1,2\%) ou por TB (26 casos $-1 \%)$. Ressalta-se que destes 13 (22\%) portadores de TB residiam em outros municípios, sendo três $(5,1 \%)$ no Estado do Amazonas, ainda que tenham sido notificados e a óbito em Porto Velho.

No mesmo período, foram notificados no SIM 28 óbitos em Porto Velho, dos quais 23 (82,1\%) apresentaram como causa básica alguma forma de TB e o restante outras causas, dentre as quais infecções múltiplas pelo HIV $(7,1 \%)$, neoplasia maligna do brônquio principal $(3,6 \%)$, outras doenças pulmonares intersticiais com fibrose $(3,6 \%)$ e complicação da gravidez, parto e puerpério pela TB (3,6\%), segundo a $10^{\mathrm{a}}$ Revisão da Classificação Estatística Internacional de Doenças e Problemas Relacionados à Saúde (CID-10) (Tabela 1) ${ }^{10}$.

Tabela 1. Distribuição dos óbitos entre os pacientes portadores de tuberculose em Porto Velho, de acordo com o Sistema de Informação de Mortalidade, no período de 2010-2015

\begin{tabular}{|c|c|c|c|}
\hline \multicolumn{2}{|r|}{ CAUSA BÁSICA } & \multicolumn{2}{|c|}{ TOTAL } \\
\hline CID-10 & Descrição & $\mathbf{N}=\mathbf{2 8}$ & $\%$ \\
\hline $\mathrm{A} 15.0$ & $\begin{array}{c}\text { TB pulmonar, com confirmação por exame microscópio de expectoração, com ou sem } \\
\text { cultura }\end{array}$ & 9 & 32,1 \\
\hline $\mathrm{A} 15.1$ & TB pulmonar, com confirmação somente por cultura & 1 & 3,6 \\
\hline A15.3 & TB pulmonar, com confirmação por meio não especificado & 6 & 21,4 \\
\hline $\mathrm{A} 15.4$ & TB dos gânglios intratorácicos, com confirmação bacteriológica e histológica & 1 & 3,6 \\
\hline A15.8 & Outras formas de TB das vias respiratórias, com confirmação bacteriológica e histológica & 1 & 3,6 \\
\hline A15.9 & TB não especificada das vias respiratórias, com confirmação bacteriológica e histológica & 5 & 17,8 \\
\hline B20.7 & Doença pelo HIV resultando em infecções múltiplas & 2 & 7,1 \\
\hline $\mathrm{C} 34.0$ & Neoplasia maligna do brônquio principal & 1 & 3,6 \\
\hline $\mathrm{J} 84.1$ & Outras doenças pulmonares intersticiais com fibrose & 1 & 3,6 \\
\hline 098.0 & TB complicando a gravidez, o parto e o puerpério & 1 & 3,6 \\
\hline
\end{tabular}

Fonte: SIM (2017)

Em relação ao perfil sociodemográfico notificado no SINAN, identificou-se que independente da causa do óbito, a média de idade foi de 46,8 anos (dp $= \pm 19,1$ anos), sendo o mínimo de 0 e máximo de 89 anos.

A maioria era do sexo masculino (66,1\%), cor/ raça autodeclarada parda $(72,9 \%)$ e residia na zona urba- na (91,5\%). Diferente das outras características, a escolaridade variou de acordo com a causa do óbito, apresentando menor escolaridade (1 a 4 anos) (38,5\%) aqueles que foram a óbito por TB e maior escolaridade ( 4 a 8 anos) (30,3\%) aqueles que foram a óbito por outras causas (Tabela 2). 
Tabela 2. Distribuição do perfil dos portadores de TB que evoluíram para o óbito durante o tratamento da doença em Porto Velho (RO), de acordo com a causa registrada no SINAN, no período de 2010-2015

\begin{tabular}{|c|c|c|c|c|c|c|c|}
\hline \multirow{3}{*}{$\begin{array}{c}\text { VARIÁVEIS } \\
\text { Sociodemográficas }\end{array}$} & \multicolumn{5}{|c|}{ CAUSAS DO ÓBITO } & \multirow{2}{*}{\multicolumn{2}{|c|}{ TOTAL }} \\
\hline & \multirow[t]{2}{*}{ TB } & \multicolumn{4}{|c|}{ NTB } & & \\
\hline & & $\mathrm{N}=26$ & $\%$ & $\mathrm{~N}=33$ & $\%$ & $\mathrm{~N}=59$ & $\%$ \\
\hline \multirow{2}{*}{ Sexo } & Masculino & 14 & 53,8 & 25 & 75,8 & 39 & 66,1 \\
\hline & Feminino & 12 & 46,2 & 8 & 24,2 & 20 & 33,9 \\
\hline \multirow{4}{*}{ Cor/Raça } & Parda & 18 & 69,3 & 25 & 75,8 & 43 & 72,9 \\
\hline & Branca & 3 & 11,5 & 5 & 15,1 & 8 & 13,5 \\
\hline & Preta & 3 & 11,5 & 3 & 9,1 & 6 & 10,2 \\
\hline & Indígena & 2 & 7,7 & 0 & 0 & 2 & 3,4 \\
\hline \multirow{7}{*}{ Escolaridade } & Analfabeto & 4 & 15,4 & 1 & 3 & 5 & 8,5 \\
\hline & De 1 a 4 anos & 10 & 38,5 & 9 & 27,3 & 19 & 32,2 \\
\hline & De 4 a 8 anos & 6 & 23,1 & 10 & 30,3 & 16 & 27,1 \\
\hline & De 8 a 11 anos & 2 & 7,7 & 6 & 18,2 & 8 & 13,5 \\
\hline & 11 anos ou mais & 0 & 0 & 2 & 6,1 & 2 & 3,4 \\
\hline & Não se aplica & 1 & 3,8 & 0 & 0 & 1 & 1,7 \\
\hline & $\begin{array}{l}\text { Ignorado/ Em } \\
\text { branco }\end{array}$ & 3 & 11,5 & 5 & 15,1 & 8 & 13,5 \\
\hline \multirow{3}{*}{ Zona } & Urbana & 24 & 92,3 & 30 & 90,9 & 54 & 91,5 \\
\hline & Rural & 2 & 7,7 & 2 & 6,1 & 4 & 6,8 \\
\hline & Periurbana & 0 & 0 & 1 & 3 & 1 & 1,7 \\
\hline
\end{tabular}

Fonte: SINAN (2017)

Em relação ao perfil clínico, observou-se que, independente da causa do óbito, a maioria era caso novo (76,3\%), forma clínica pulmonar (78\%), sem doenças e agravos associados, tais como etilismo $(86,4 \%)$ e diabetes (79,7\%), com raio-X de tórax suspeito de TB $(91,5 \%)$ e não acompanhado pelo regime do TDO (74,6\%) (Tabela 3).

Os portadores de TB que foram a óbito pela doença não possuíam aids (50\%), apresentaram teste anti-HIV negativo $(46,2 \%)$ e baciloscopia de escarro positiva (42,3\%). Enquanto os portadores de TB que foram a óbito por outras causas possuíam aids (45,5\%), teste anti-HIV positivo $(45,5 \%)$ e baciloscopia de escarro negativa (48,5\%). Além disso, observou-se que o tabagismo foi ignorado ou estava em branco em sua maioria $(78,0 \%)$ (Tabela 3).
No que concerne aos exames de controle mensal, independente da causa do óbito, houve aumento progressivo de registros em branco durante o tratamento dos doentes de TB, sendo no primeiro mês de $47,4 \%$ e no sexto mês de 91,5\% (Tabela 4). 
Tabela 3. Distribuição do perfil clínico dos portadores de TB que evoluíram para o óbito durante o tratamento da doença em Porto Velho (RO), de acordo com a causa registrada no SINAN, no período de 2010-2015

\begin{tabular}{|c|c|c|c|c|c|c|c|c|}
\hline VAR & VEIS & & & AUSAS & ÓBITO & & & \\
\hline & & & & & & & & \\
\hline Clínicas & & & $\mathrm{N}=26$ & $\%$ & $\mathrm{~N}=33$ & $\%$ & $\mathrm{~N}=59$ & $\%$ \\
\hline & & Novo & 21 & 80,9 & 24 & 72,8 & 45 & 76,3 \\
\hline Tino de coso & & Recidiva & 3 & 11,5 & 4 & 12,1 & 7 & 11,9 \\
\hline 11" & & ingresso após abandono & 1 & 3,8 & 5 & 15,1 & 6 & 10,1 \\
\hline & & Transferência & 1 & 3,8 & 0 & 0 & 1 & 1,7 \\
\hline & & Pulmonar & 22 & 84,7 & 24 & 72,8 & 46 & 78 \\
\hline & & Extrapulmonar* & 3 & 11,5 & 8 & 24,2 & 11 & 18,6 \\
\hline Forma clínica & & $\begin{array}{c}\text { Pulmonar + } \\
\text { Extrapulmonar* }\end{array}$ & 1 & 3,8 & 1 & 3 & 2 & 3,4 \\
\hline & & Não & 13 & 50 & 11 & 33,3 & 24 & 40,7 \\
\hline & 旁 & Sim & 5 & 19,2 & 15 & 45,5 & 20 & 33,9 \\
\hline & & Ignorado/Em branco & 8 & 30,8 & 7 & 21,2 & 15 & 25,4 \\
\hline & き & Não & 23 & 88,5 & 28 & 84,9 & 51 & 86,4 \\
\hline & : & $\operatorname{Sim}$ & 3 & 11,5 & 4 & 12,1 & 7 & 11,9 \\
\hline & & Ignorado/ Em branco & 0 & 0 & 1 & 3 & 1 & 1,7 \\
\hline Doencas e agravos associados & & Não & 3 & 11,5 & 8 & 24,2 & 11 & 18,6 \\
\hline & 莺 & $\operatorname{Sim}$ & 0 & 0 & 2 & 6,1 & 2 & 3,4 \\
\hline & है & Ignorado/Em branco & 23 & 88,5 & 23 & 69,7 & 46 & 78 \\
\hline & $\approx$ & Não & 19 & 73,1 & 28 & 84,9 & 47 & 79,7 \\
\hline & $\begin{array}{l}\stackrel{\mathscr{U}}{\pi} \\
\frac{\pi}{\pi}\end{array}$ & Sim & 7 & 26,9 & 4 & 12,1 & 11 & 18,6 \\
\hline & & Ignorado & 0 & 0 & 1 & 3 & 1 & 1,7 \\
\hline Exames diagnósticos & & & & & & & & \\
\hline & & Positiva & 11 & 42,3 & 11 & 33,3 & 22 & 37,3 \\
\hline Baciloscopia de escarro & & Negativa & 11 & 42,3 & 16 & 48,5 & 27 & 45,8 \\
\hline & & Não realizada & 4 & 15,4 & 6 & 18,2 & 10 & 16,9 \\
\hline & & Suspeito & 25 & 96,2 & 29 & 87,8 & 54 & 91,5 \\
\hline Raio-X de tórax & & Não realizado & 1 & 3,8 & 2 & 6,1 & 3 & 5,1 \\
\hline & & Outra patologia & 0 & 0 & 2 & 6,1 & 2 & 3,4 \\
\hline & & Negativo & 12 & 46,2 & 11 & 33,3 & 23 & 39 \\
\hline Teste Anti-HIV & & Positivo & 5 & 19,2 & 15 & 45,5 & 20 & 33,9 \\
\hline & & Não realizado & 9 & 34,6 & 7 & 21,2 & 16 & 27,1 \\
\hline Tratamento & & & & & & & & \\
\hline & & Não & 19 & 73,1 & 25 & 75,8 & 44 & 74,6 \\
\hline TDO & & Sim & 5 & 19,2 & 4 & 12,1 & 9 & 15,2 \\
\hline & & Em branco & 2 & 7,7 & 4 & 12,1 & 6 & 10,2 \\
\hline
\end{tabular}

*Forma clínica extrapulmonar e pulmonar + extrapulmonar: pleural $(69,2 \%)$, miliar (15,4\%), ganglionar periférica $(7,7 \%)$ ou outra $(7,7 \%)$. Fonte: SINAN (2017) 
Tabela 4. Distribuição dos exames de controle mensal realizado pelos portadores de TB que foram a óbito durante o tratamento, de acordo com a causa do óbito no SINAN em Porto Velho, no período de 2010-2015

\begin{tabular}{|c|c|c|c|c|c|c|c|}
\hline \multirow[t]{2}{*}{ VARIÁVEIS } & \multirow{3}{*}{ TB } & \multicolumn{4}{|c|}{ CAUSAS DO ÓBITO } & \multirow{2}{*}{\multicolumn{2}{|c|}{ TOTAL }} \\
\hline & & & & & & & \\
\hline Exames de controle mensal & & $\mathrm{N}=26$ & $\%$ & $\mathrm{~N}=33$ & $\%$ & $\mathrm{~N}=59$ & $\%$ \\
\hline \multirow{5}{*}{ Baciloscopia $1^{\circ}$ mês } & Não realizada & 10 & 38,5 & 12 & 36,4 & 22 & 37,3 \\
\hline & Positiva & 1 & 3,8 & 1 & 3 & 2 & 3,4 \\
\hline & Negativa & 0 & 0 & 3 & 9,1 & 3 & 5,1 \\
\hline & Não se aplica & 0 & 0 & 4 & 12,1 & 4 & 6,8 \\
\hline & Em branco & 15 & 57,7 & 13 & 39,4 & 28 & 47,4 \\
\hline \multirow{4}{*}{ Baciloscopia $2^{\circ}$ mês } & Não realizada & 8 & 30,8 & 13 & 39,4 & 21 & 35,6 \\
\hline & Negativa & 1 & 3,8 & 3 & 9,1 & 4 & 6,8 \\
\hline & Não se aplica & 0 & 0 & 4 & 12,1 & 4 & 6,8 \\
\hline & Em branco & 17 & 65,4 & 13 & 39,4 & 30 & 50,8 \\
\hline \multirow{5}{*}{ Baciloscopia $3^{\circ}$ mês } & Não realizada & 8 & 30,8 & 11 & 33,3 & 19 & 32,2 \\
\hline & Positiva & 0 & 0 & 1 & 3 & 1 & 1,7 \\
\hline & Negativa & 0 & 0 & 3 & 9,1 & 3 & 5,1 \\
\hline & Não se aplica & 0 & 0 & 4 & 12,1 & 4 & 6,8 \\
\hline & Em branco & 18 & 69,3 & 14 & 42,5 & 32 & 54,2 \\
\hline \multirow{4}{*}{ Baciloscopia $4^{\circ}$ mês } & Não realizada & 3 & 11,5 & 7 & 21,2 & 10 & 16,9 \\
\hline & Negativa & 0 & 0 & 2 & 6,1 & 2 & 3,4 \\
\hline & Não se aplica & 0 & 0 & 4 & 12,1 & 4 & 6,8 \\
\hline & Em branco & 23 & 88,5 & 20 & 60,6 & 43 & 72,9 \\
\hline \multirow[t]{4}{*}{ Baciloscopia $5^{\circ}$ mês } & Não realizada & 2 & 7,7 & 1 & 3 & 3 & 5,1 \\
\hline & Negativa & 0 & 0 & 1 & 3 & 1 & 1,7 \\
\hline & Não se aplica & 0 & 0 & 4 & 12,1 & 4 & 6,8 \\
\hline & Em branco & 24 & 92,3 & 27 & 81,9 & 51 & 86,4 \\
\hline \multirow{3}{*}{ Baciloscopia $6^{\circ}$ mês } & Não realizada & 0 & 0 & 1 & 3 & 1 & 1,7 \\
\hline & Não se aplica & 0 & 0 & 4 & 12,1 & 4 & 6,8 \\
\hline & Em branco & 26 & 100 & 28 & 84,9 & 54 & 91,5 \\
\hline
\end{tabular}

Fonte: SINAN (2017)

\section{DISCUSSÃO}

\section{A RELAÇÃO ENTRE O SINAM E O SIM}

Ainda que a Declaração de Óbito (DO) apresente o motivo que ocasionou o mesmo, não deixa claro se a causa básica está associada, impossibilitando a avaliação dos fatores de risco que desencadearam tal desfecho ${ }^{3}$, como ocorreu neste estudo, no qual não foi possível saber a contribuição da TB no óbito, considerando que por inúmeras vezes a doença aparece apenas como causa associada.
Dos 28 óbitos notificados no SIM, apenas o caso de complicação na gravidez, parto e puerpério estava notificado também no SINAN. Neste sentido, os diversos sistemas de informação em saúde, com diferentes especificidades e não compatíveis entre si, podem gerar déficit ou duplicidade de dados, e consequentemente interferir nas ações de controle da doença e no processo de tomada de decisãa ${ }^{11}$.

Um portador de TB que evolui para óbito sem notificação no SINAN torna-se desconhecido para o PNCT e para o desenvolvimento das ações de vigilância em saúde pela falta de integração entre os sistemas de informa- 
ção. Além disso, pode-se supor que os contatos desse caso não foram examinados, o que agrava o problema $^{12}$.

O pós-óbito classifica os casos que nunca foram registrados no SINAN, sendo notificados após a morte, em decorrência da investigação epidemiológica, refletindo em falhas na busca de sintomáticos respiratórios e na oferta da assistência prestada. Em 2016, 517 casos foram classificados como pós-óbito no Brasil, o que pode ser reflexo das atividades de vigilância do óbito por TB como causa básica ou associada ${ }^{7}$.

\section{PERFIL SOCIODEMOGRÁFICO}

Homens, em idade economicamente ativa, estão mais expostos ao óbito relacionado ao M. tuberculosis, considerando que a procura pelo serviço de saúde ocorre, principalmente, diante da gravidade dos sintomas. Isso se deve ao distanciamento compulsório das atividades laborais, comprometimento da renda familiar e estigmatização social causada pelo adoecimento ${ }^{13}$.

Horários incompatíveis, demora no atendimento, atrasos e faltas no trabalho para o comparecimento em consultas médicas, podem distanciar os casos suspeitos dos serviços de saúde, por receio de perder o emprego. O que também dificulta o diagnóstico precoce da TB e favorece outros desfechos, tais como o abandono e o óbito ${ }^{14}$.

A maioria autodeclarada parda justifica-se por esta ser a cor/raça predominante no município, pelo processo de colonização do Estado. Considerando que centros urbanos e aglomerados populacionais predispõem a transmissão da TB, observou-se que a zona de residência urbana foi predominante ${ }^{4}$.

A baixa escolaridade foi observada independente da causa de óbito, entretanto, aqueles que foram a óbito pela doença apresentaram a escolaridade menor do que aqueles que foram a óbito por outras causas. $\mathrm{O}$ nível de instrução influencia no entendimento acerca da doença ${ }^{13}$ e se configura como um fator que auxilia na adesão e sucesso do tratamento ${ }^{15}$.

\section{PERFIL CLÍNICO}

A proporção de casos novos está associada a forma clínica pulmonar, a qual é a mais frequente e responsável pela cadeia de transmissão da doença. Em um ano, cada doente bacilífero é capaz de infectar de 10 a 15 pessoas, cerca de $5 \%$ a $10 \%$ das pessoas infectadas desenvolverão a forma clínica da doença, já que esta depende da imunidade do indivíduo ${ }^{16}$, dentre outros fatores.

Não foi observado, independente da causa do óbito, etilismo e diabetes na maioria dos casos, apesar de serem agravos associados relevantes na literatura ${ }^{17,18}$. No entanto, é possível uma reflexão sobre a forma como é realizada a coleta e registro na ficha de notificação, seja pela associação entre consumo de álcool dentre os portadores de TB e o maior risco de obterem desfechos desfavoráveis, quanto pelo conhecimento de que este consumo pode causar potenciais efeitos hepatotóxicos nocivos quando concomitante com as drogas antituberculose ${ }^{17}$.

A relação entre o aumento da mortalidade dentre os portadores de TB e diabetes é inversamente proporcional, ainda que os pacientes com diabetes apresentem manifestações clínicas mais graves, maior propensão à falha terapêutica, recorrência e recidiva da $\mathrm{TB}^{18}$. Entretanto, por se tratar da comorbidade de duas condições crônicas, podemos inferir sobre a corresponsabilização do usuário e o acompanhamento longitudinal dos profissionais de saúde para a oferta do cuidado em sua integralidade.

$\mathrm{O}$ raio-X de tórax é um exame de imagem que pode ser solicitado para elucidação diagnóstica juntamente com a baciloscopia de escarro e a avaliação clínica. Tal achado também pode estar associado ao tipo de serviço de saúde procurado pelo doente (geralmente, atenção terciária), a baixa suspeita de TB e sensibilização pelos profissionais de saúde para solicitar outro exame, como a baciloscopia de escarro, ${ }^{(14)}$ a qual era referência para o diagnóstico da TB até 2015.

Atualmente, o Teste Rápido Molecular para TB (TRM-TB) é preconizado por apresentar sensibilidade de aproximadamente $90 \%$ (enquanto a da baciloscopia de escarro era de $65 \%$ ) e especificidade de $99 \%{ }^{19,20}$. O diagnóstico por meio do TRM-TB ocorre em menor tempo, indica a existência de resistência a principal droga (rifampicina) do esquema básico do tratamento, favorecendo seu início oportuno e diminuindo o tempo necessário para o início da terapia com medicamento de segunda linha, se necessário ${ }^{19,20}$.

O TDO consiste na supervisão da ingesta medicamentosa por um profissional ou familiar capacitado, 
visando garantir a adesão e cura do tratamento. Apesar disso, observou-se que, independente da causa do óbito, poucos casos estavam sendo tratados sob o regime do TDO, ainda que tal regime possa reduzir em $40 \%$ a mortalidade quando comparado com aqueles que realizaram o autoadministrado ${ }^{21}$.

A baixa cobertura do TDO é uma realidade do município, na qual os profissionais de saúde pouco/raramente se apropriam desta ferramenta para o controle e tratamento dos casos, tendo em vista o papel da APS, muitas vezes visto e tido como modelo coadjuvante na assistência e ações de vigilância em saúde, principalmente na TB.

A aids e o teste anti-HIV variaram de acordo com a causa do óbito, apresentando maior negatividade nos óbitos por TB e positividade nos óbitos por NTB. Um dos principais fatores de risco para TB é a infecção pelo HIV, considerando que as pessoas que vivem com HIV/ aids (PVHA) apresentam de 21 a 34 vezes mais chance de desenvolver TB ativa em comparação com a população geral, além de ser a condição que causa o maior impacto nos óbitos por aids e $\mathrm{TB}^{22}$.

O teste anti-HIV está incluído entre os exames diagnósticos da TB para o rastreamento da coinfecção TB/HIV. A terapia isolada para o HIV não garante a prevenção da coinfecção TB/HIV. Por isso, deve-se atuar no cuidado de forma holística, considerando as vulnerabilidades socioeconômicas e culturais do indivíduo ${ }^{22}$.

Quanto ao acompanhamento, os exames de controle mensal são importantes para a avaliação da eficácia do tratamento. Apesar disso, a não realização e/ou registro das baciloscopias para controle mensal dos portadores de TB também é verificada na literatura ${ }^{23}$, representando falhas no acompanhamento dos casos.

A mediana do tempo de tratamento foi de 70,5 dias, sendo o mínimo de um e máximo de 402 dias. Ou seja, a maioria dos óbitos ocorreu antes da conclusão do terceiro mês de tratamento, o que remete a gravidade dos sinais e sintomas, bem como o retardo no diagnóstico e falhas no acompanhamento dos casos ${ }^{3}$.

\section{CONCLUSÃO}

Diante de tais achados, verificou-se que independente da causa do óbito, o perfil se configura por ho- mens, em idade economicamente ativa, de cor/raça autodeclarada parda, residindo na zona urbana, caso novo, forma clínica pulmonar, não etilista, não diabético, com raio-X de tórax suspeito de TB e não acompanhado pelo TDO.

Diferente das características citadas, escolaridade, aids, baciloscopia de escarro e teste anti-HIV variaram de acordo com a causa do óbito, enquanto o tabagismo foi ignorado ou estava em branco em sua maioria, assim como as baciloscopias de controle mensal.

Além disso, verificou-se que os portadores de TB que foram a óbito pela doença possuíam baciloscopia de escarro positiva, enquanto que aqueles que foram a óbito por outras causas eram coinfectados TB/HIV. Estes aspectos refletem na gravidade dos sinais e sintomas daqueles que tinham a forma clínica pulmonar, bem como possíveis complicações do HIV ou não adesão ao tratamento.

A baixa completude dos dados sobre tabagismo e baciloscopias de controle mensal, bem como a qualidade dos registros, incluindo as divergências entre os sistemas de informação, se configuram como fatores limitantes do presente estudo.

Neste sentido, ressalta-se a importância da integração entre o SINAN e SIM pela vigilância do óbito, como também a qualificação profissional para o preenchimento adequado das informações, tendo em vista a sua relevância no reconhecimento das deficiências que prejudicam o controle da TB e favorecem o óbito, nos diversos âmbitos da assistência à saúde.

\section{AGRADECIMENTOS}

Ao Conselho Nacional de Desenvolvimento Científico e Tecnológico (CNPq), pelo financiamento por meio de bolsas de iniciação científica.

\section{REFERÊNCIAS}

1. World Health Organization. Global tuberculosis report 2016. Geneva: World Health Organization; 2016. Disponível em: http://apps.who.int/medicinedocs/en/d/ Js23098en/

2. World Health Organization. The End TB strategy. Ge- 
neva: World Health Organization; 2015. Disponível em: http://www.who.int/tb/post2015_strategy/en/

3. Rocha MS, Oliveira GP, Aguiar FP, Saraceni V, Pinheiro RJ. Do que morrem os pacientes com tuberculose: causas múltiplas de morte de uma coorte de casos notificados e uma proposta de investigação de causas presumíveis. Cad. Saúde Pública. 2015; 31(4): 709-21. Disponível em: http://dx.doi.org/10.1590/ 0102-311X00101214

4. Ceccon RF, Maffacciolli R, Burille A, Meneghel SN, Oliveira DLLC, Gerhardt TE. Mortalidade por tuberculose nas capitais brasileiras, 2008-2010. Epidemiol. Serv. Saúde. 2017; 26(2): 349-58. Disponível em: http://dx.doi.org/10.5123/s1679-49742017000200012

5. Perrechi MCT, Ribeiro AS. Desfechos de tratamento de tuberculose em pacientes hospitalizados e não hospitalizados no município de São Paulo. J bras. pneumol. 2011; 37(6): 783-90. Disponível em: http://dx.doi. org/10.1590/S1806-37132011000600012

6. Ministério da Saúde (BR). Secretaria de Vigilância em Saúde. Boletim Epidemiológico. Perspectivas brasileiras para o fim da tuberculose como problema de saúde pública. Brasília (DF): Ministério da Saúde; 2016. Disponível em: http://portalarquivos2 saude. gov.br/images/pdf/2016/marco/24/2016-009-Tuberculose-001.pdf

7. Ministério da Saúde (BR). Secretaria de Vigilância em Saúde. Boletim Epidemiológico. Indicadores prioritários para o monitoramento do Plano Nacional pelo Fim da tuberculose como problema de saúde pública no Brasil. Brasília (DF): Ministério da Saúde; 2017a.

8. Ministério da Saúde (BR). Secretaria de Vigilância em Saúde. Departamento de Vigilância das Doenças Transmissíveis. Panorama da tuberculose no Brasil: indicadores epidemiológicos e operacionais. Brasília (DF): Ministério da Saúde; 2014a. Disponível em: http://bvsms.saude.gov.br/bvs/publicacoes/panorama\%20tuberculose\%20brasil_2014.pdf

9. Brasil. Resolução 466, de 12 de dezembro de 2012. Aprova diretrizes e normas regulamentadoras de pesquisas envolvendo seres humanos. Diário Oficial da União, Brasília, 12 dez. 2012. Disponível em: http://bvsms.saude.gov.br/bvs/saudelegis/cns/2013/ res0466_12_12_2012.html

10. Organização Mundial da Saúde. Classificação Estatística Internacional de Doenças e Problemas Rela- cionados à Saúde - CID-10. Organização Mundial da Saúde; 2008. Disponível em: http://apps.who.int/classifications/icd10/browse/2016/en

11. Orfão NH, Crepaldi NY, Brunello MEF, Andrade RLP, Monroe AA, Ruffino-Netto A et al. Coordenação da assistência à tuberculose: registro de dados e a implementação de um sistema informatizado. Ciênc. saúde coletiva. 2017; 22(6): 1969-77. Disponível em: http:// dx.doi.org/10.1590/1413-81232017226.15352016

12. Ministério da Saúde (BR). Secretaria de Vigilância em Saúde. Departamento de Vigilância das Doenças Transmissíveis. Protocolo de vigilância do óbito com menção de tuberculose nas causas de morte. Brasília (DF): Ministério da Saúde; 2017b. Disponível em: http://dive.sc.gov.br/conteudos/publicacoes/Protocolo-para-Vigilncia-do-obito.pdf

13. Cavalcante EFO, Silva DMGV. Perfil de pessoas acometidas por tuberculose. Rev Rene. 2013; 14(4): 72029. Disponível em: http://dx.doi.org/10.15253/rev\%20 rene.v14i4.3531

14. Loureiro RB, Villa TCS, Ruffino-Netto A, Peres RL, Braga JU, Zandonade $\mathrm{E}$ et al. Acesso ao diagnóstico da tuberculose em serviços de saúde do município de Vitória, ES, Brasil. Ciênc. saúde coletiva. 2014; 19(4): 1233-44. Disponível em: http://dx.doi. org/10.1590/1413-81232014194.01002013

15. Orfão NH, Andrade RLP, Beraldo AA, Brunello MEF, Scatena LM, Villa TCS. Adesão terapêutica ao tratamento da tuberculose em um município do estado de São Paulo. Cienc Cuid Saude. 2015; 14(4): 1453-61. Disponível em: http://dx.doi.org/10.4025/cienccuidsaude.v14i4.25093

16. Barbosa DRM, Almeida MG, Martins LM, Silva TMGV, Pedrosa JIS, Barbosa MM. Aspectos socioculturais da tuberculose e diálogo com políticas públicas em saúde no Brasil. Rev. Gestão \& Saúde. 2013; edição especial: 1857-67. Disponível em: http://dx.doi.org/10.18673/ gs.v1i1.22983

17. Duraisamy K, Mrithyunjayan S, Ghosh S, Nair SA, Balakrishnan S, Subramoniapillai J et al. Does Alcohol Consumption during Multidrug-resistant Tuberculosis Treatment Affect Outcome? A Population-based Study in Kerala, India. Ann Am Thorac Soc. 2014; 11(5): 712-18. Disponível em: https://www.ncbi.nlm. nih.gov/pmc/articles/PMC4631605/pdf/nihms731743. pdf 
18. Corona MEJ, Hervert LPC, García LG, Reyes LF, Sánchez GD, Valle MB et al. Association of diabetes and tuberculosis: impact on treatment and post-treatment outcomes. Thorax. 2013; 68(3): 214-20. Disponível em: https:/www.ncbi.nlm.nih.gov/pmc/articles/ PMC3585483/pdf/thoraxjnl-2012-201756.pdf

19. Ministério da Saúde (BR). Secretaria de Vigilância em Saúde. Boletim Epidemiológico. O controle da tuberculose no Brasil: avanços, inovações e desafios. Brasília (DF): Ministério da Saúde; 2014b. Disponível em: http:/bvsms.saude.gov.br/bvs/periodicos/boletim_epidemiologico_numero_2_2014.pdf

20. Souza KMJ, Sá LD, Silva LMC, Palha PF. Atuação da Enfermagem na transferência da política do tratamento diretamente observado da tuberculose. Rev Esc Enferm USP. 2014; 48(5): 874-82. Disponível em: http://www.scielo.br/pdf/reeusp/v48n5/pt_00806234-reeusp-48-05-874.pdf

21. Yen YF, Rodwell TC, Yen MY, Shih HC, Hu BS, Li LH et al. DOT associated with reduced all-cause mortality among tuberculosis patients in Taipei, Taiwan, 20062008. Int J Tuberc Lung Dis. 2012; 16(2): 178-84. Disponível em: https://www.ncbi.nlm.nih.gov/pmc/ articles/PMC3289585/pdf/nihms355804.pdf

22. Magnabosco GT, Lopes LM, Andrade RLP, Brunello MEF, Monroe AA, Villa, TCS. Controle da tuberculose em pessoas vivendo com HIV/aids. Rev. Latino-Am. Enfermagem. 2016; 24: e2798. Disponível em: http:// dx.doi.org/10.1590/1518-8345.1187.2798

23. Angelotti LCZ, Alexandre PBD, Miranzi SSC, Scatena, LM. Qualidade de dados de notificação e acompanhamento dos casos de tuberculose em Minas Gerais. REAS. 2013; 2(2): 84-98. Disponível em: http://seer. uftm.edu.br/revistaeletronica/index.php/enfer/article/ view/387/410

Recebido em: 25/06/2018

Aceito em: 08/08/2018 